\title{
The prokineticin Bv8 sensitizes cutaneous terminals of female mice to heat
}

\author{
T. Hoffmann ${ }^{1}$, L. Negri ${ }^{2}$, D. Maftei ${ }^{2}$, R. Lattanzi ${ }^{2}$, P.W. Reeh ${ }^{1}$ \\ 1 Institute for Physiology and Pathophysiology, University of Erlangen-Nuremberg, Germany \\ 2 Department of Physiology and Pharmacology, Sapienza University of Rome, Italy
}

\begin{abstract}
Correspondence
Tali Hoffmann

E-mail: tal.hoffmann@fau.de

Funding sources

This article was funded by the Deutsche Forschungsgemeinschaft, grant RE 704/2-1 56874 and the Sapienza University of Rome.
\end{abstract}

Conflicts of interest

None declared.

Accepted for publication

17 December 2015

doi:10.1002/ejp.857

\begin{abstract}
Background: Injection of the noxious peptide Bv8 has previously been shown to induce a biphasic thermal hyperalgesia in rodents, the first peak presumably due to peripheral sensitization. This hypothesis has never been directly confirmed. We have assessed whether Bv8 can indeed sensitize peripheral nerve fibres in the mouse to heat.

Methods: We used recordings from single cutaneous fibres, cutaneous calcitonin gene-related peptide (CGRP) release and immunostaining in nerves and plantar skin to evaluate the Bv8 effects on cutaneous nerves. Results: Application of Bv8 at nanomolar concentrations (30$310 \mathrm{nmol} / \mathrm{L}$ ) to skin preparations significantly increased the heatinduced discharge, the heat-induced afterdischarge and reduced threshold temperature of single unmyelinated polymodal fibres. Furthermore, application of Bv8 to hind-paw skin flaps or trigeminal ganglia significantly elevated their heat-induced CGRP release. Capsaicin-induced and to a lesser extent also $\mathrm{KCl}$-induced CGRP releases were also augmented after Bv8 treatment. Immunohistochemistry revealed co-localization of prokineticin 2 (Bv8 ortholog in rodents) and CGRP in both plantar skin and nerve tissues. These results confirm that Bv8 sensitizes cutaneous nerve endings to heat, partly, although not exclusively through TRPVl activation.

Conclusion: Our results thus support the hypothesis that the first hyperalgesic phase to follow Bv8 injection to hind paws of intact animals is due to peripheral sensitization of nociceptors.

What does this study add?: Our data provide mechanistic insights into the effect Bv8 application exerts on afferent nerve endings and into the concomitant development of thermal hyperalgesia.
\end{abstract}

\section{Introduction}

Bv8 is a small 8-kDa peptide isolated from the skin of the frog Bombina variegata (Mollay et al., 1999). It has similar structure to protein $\mathrm{A}$, present in the venom of Dendroaspis polylepis [the black mamba (Joubert and Strydom, 1980)]. Bv8 homologs and orthologs were found in fish, reptiles, mouse, rat, cattle, monkey and man [see (Negri et al., 2007) for summary]. The mammalian orthologs of Bv8, named prokineticin 1 (PK1) and prokineticin 2
(PK2), as well as purified Bv8 bind to the G-protein-coupled receptors prokineticin receptor 1 (PKR1) and prokineticin receptor 2 [PKR2 (Lin et al., 2002; Soga et al., 2002)]. PKR1 and PKR2 are expressed in various endocrine glands, the gastrointestinal tract, prostate, spleen, pancreas, brain, spinal cord and dorsal root ganglia [DRG (Lin et al., 2002; Negri et al., 2006b)], consisting with suggested involvement in regulating neuronal survival, circadian rhythms, angiogenesis, hematopoiesis and the reproductive cycle (Wechselberger et al., 1999; 
LeCouter et al., 2001, 2004; Melchiorri et al., 2001; Cheng et al., 2002; LeCouter and Ferrara, 2003). Both PK receptors are expressed in mouse DRG neurons, also in small peptidergic DRGs co-expressing the transient receptor potential vanilloid receptor 1 [TRPVl (Vellani et al., 2006)]. The neuronal projections of such peptidergic cells innervate many tissues and participate in nociceptive processes (Immke and Gavva, 2006). Accordingly, Bv8 and PKRs have previously been connected to nociception and inflammatory pain. Behavioural tests in rats show that systemic injection of Bv8 or potent analogues induces a biphasic thermal hyperalgesia and tactile allodynia (Negri et al., 2002, 2005; Vellani et al., 2006). It has been suggested, although never proved, that the initial phase is due to a local activation of skin-nerve endings, while the latter action is induced through central sensitization. Bv8 was also shown to sensitize TRPVl in DRG cultures from these rats through activation of protein kinase C epsilon [PKCE (Vellani et al., 2006)]. In addition, behavioural tests in mice lacking PKRl showed impaired response to noxious heat and capsaicin/ acetic acid/complete Freund's adjuvant (CFA) injection (Negri et al., 2006a). In another study, blocking PKRs alleviated thermal hyperalgesia and tactile allodynia in mice following chronic constriction of sciatic nerve (Maftei et al., 2014). While the involvement of the PK receptors in the development of peripheral neuronal sensitization under inflammatory/neuropathic conditions or Bv8 treatment is well established, further insights concerning mechanisms of peripheral activation remain unresolved. In this study, we evaluate the possibility that PKR-mediated cutaneous sensitization occurs through direct activation of nociceptive endings. For this, we record the electrical discharge from cutaneous single afferent units, as well as measure the release of the neuropeptide calcitonin gene-related peptide (CGRP) following Bv8 application. The latter is an established technique for studying mass activation of primary afferents, which have been reported to release neuropeptides from the soma as well as axons (Levine et al., 1993; Sauer et al., 1999; Eberhardt et al., 2008).

\section{Methods}

\subsection{Animals}

The study was performed on male and female inbred C57BL/6 mice weighing 20-25 g. Mice were housed in group cages in a temperature-controlled environment with a 12-h light-dark cycle and were supplied with food and water ad libitum. Animals were killed in a pure $\mathrm{CO}_{2}$ atmosphere.

\subsection{Immunofluorescence}

Sciatic nerve and plantar skin were dissected from transcardially perfused (PBS followed by $4 \%$ paraformaldehyde) mice, embedded in cryostat medium and frozen. Serial sections $(20 \mu \mathrm{m})$ were cut using a cryostat and thaw-mounted onto glass slides. Prior to immunofluorescence staining, all sections were blocked with 3\% normal donkey serum (serum source was the same as the secondary antibody producing species), containing $0.3 \%$ Triton X-100 for $\mathrm{l} \mathrm{h}$ at room temperature. Subsequently, sections were incubated at $4{ }^{\circ} \mathrm{C}$ overnight with primary antibodies diluted in PBS-0.3\% Triton X-100: 1/200 rabbit polyclonal anti-prokineticin 2 (PKR2; AbCam, Cambridge, UK) and 1/300 goat polyclonal antiCGRP (AbCam). The sections were then incubated for $2 \mathrm{~h}$ at room temperature in $1 / 200$ antispecies $\operatorname{IgG}$ antibodies coupled to Alexa Fluor-488 or 555 (Immunological Sciences, Rome, Italy). Nuclei were stained with DAPI 1/500. The stained sections were examined using a confocal laser scanning microscope (Leica SP5; Leica Microsystems, Wetzlar, Germany).

\subsection{CGRP release}

Flaps of the hairy skin from both lower legs and feet were excised and wrapped around acrylic glass rods (6 mm diameter) with the corium side exposed as previously described (Sauer et al., 1998). Both samples were processed in parallel and placed in glass tubes containing $200 \mu \mathrm{L}$ SIF solution [synthetic interstitial fluid (SIF) (Bretag, 1969) consisting (in $\mathrm{mmol} / \mathrm{L})$ of $107.8 \mathrm{NaCl}, 26.2 \mathrm{NaCO}_{3}, 9.64 \mathrm{Na}$-gluconate, 7.6 sucrose, 5.05 glucose, $3.48 \mathrm{KCl}, 1.67$ $\mathrm{NaH}_{2} \mathrm{PO}_{4}, 1.53 \mathrm{CaCl}_{2}$ and $0.69 \mathrm{MgSO}_{4}$, continuously gassed with carbogen (95\% oxygen and 5\% carbon dioxide) equilibrating the solution at $\mathrm{pH}$ 7.4] and positioned in a shaking bath set to $32{ }^{\circ} \mathrm{C}$ for a washout period of $30 \mathrm{~min}$. Skin flaps were then consecutively passed through a set of glass tubes, each incubation step lasting $5 \mathrm{~min}$. The first incubation step was to determine basal CGRP release and the reaction tube contained only SIF solution. The second tubes contained either SIF or SIF with Bv8 (10, 100 or $310 \mathrm{nmol} / \mathrm{L})$ to assess potential CGRP-releasing effects of Bv8. The third incubation step assessed heat-induced CGRP release and a potential sensitizing effect of Bv8; reaction tubes contained either SIF or SIF + Bv8 and were positioned in a shaking bath set to $47^{\circ} \mathrm{C}$. The fourth incubation step assessed 
recovery of the response and was measured in reaction tubes containing SIF solution at $32{ }^{\circ} \mathrm{C}$.

For trigeminal ganglion (TG) experiments, ganglia were excised from female mice as previously described (Eberhardt et al., 2008). TG preparations were then immersed in SIF solution in eppendorf cups for a washout period of $30 \mathrm{~min}$ at $37{ }^{\circ} \mathrm{C}$. Subsequently, the TGs were subjected to a series of four incubation steps by sampling and replacing the SIF in the cups without touching the ganglia. Each incubation lasted $5 \mathrm{~min}$ and the protocol paralleled that of the skin experiments with the 3rd step acting as stimulus $\left(47^{\circ} \mathrm{C}\right.$ or $\left.\mathrm{KCl} 60 \mathrm{mmol} / \mathrm{L}\right)$. Calcitonin gene-related peptide (CGRP) baseline measurements in the ganglia were performed at $37^{\circ} \mathrm{C}$.

CGRP levels contained in the incubation fluid of both skin and ganglia preparations were measured and analysed using commercial enzyme immunoassays (EIAs; SPIbio, Moutigny, France), as previously described (Averbeck and Reeh, 2001). For Bv8 pretreatment experiments, skin flaps were incubated during a prolonged washout period either in SIF or in SIF + Bv8 (5 nmol/L) for $90 \mathrm{~min}$. Subsequently, skin flaps were rinsed twice in SIF and passed through similar experimental steps as described above except for the third tube containing capsaicin $(1 \mu \mathrm{mol} / \mathrm{L})$ at $32{ }^{\circ} \mathrm{C}$ for stimulation.

\subsection{Single-fibre recordings}

Based on the CGRP release results, only female C57BL/6 mice were used for the single-fibre recordings. These were obtained from mechanoreceptive Cfibres of the saphenous nerve using the isolated skinnerve preparation as previously described (Reeh, 1986). Briefly, a flap of the hind-paw hairy skin still attached to the innervating saphenous nerve was excised and fixed in an organ bath, corium side up. The chamber containing the skin tissue was continuously superfused with SIF solution, ambient temperature was maintained at $32{ }^{\circ} \mathrm{C}$ and $\mathrm{pH}$ was adjusted to 7.4 through carbogen gassing. The cut nerve trunk was placed on a mirror in a separate chamber where SIF was overlaid with paraffin oil, desheathed and divided under microscopic control. For each recorded nerve fibre, a mechanoreceptive field (RF) was mapped using a blunt glass rod. Electrostimulation was then applied to the RF through a metal microelectrode to assess the conduction velocity of the fibre. The marking technique combining electrical and mechanical stimulation of the RF was used (Schmelz et al., 1995) to ensure that a distinct single-fibre was recorded from. The mechanical threshold of the fibre was determined using calibrated 15 von Frey bristles ranged $1-128 \mathrm{mN}$ in a geometric scale. Subsequently, isolating steel ring ( $9 \mathrm{~mm}$ diameter) was placed around the RF, evacuated from fluid, and radiant heat (20 s ramp of $32^{\circ}-48{ }^{\circ} \mathrm{C}$ ) as well as cold (by superfusion of ice-cold buffer) stimulations were applied. The same steel ring chamber was also used to apply chemicals to the RF at the well permeable corium side of the skin.

For acute Bv8 administration, solutions (in SIF) of 10 , 30 or $100 \mathrm{nmol} / \mathrm{L}$ were applied to the heatresponsive unmyelinated nerve fibres $(\mathrm{CMH})$ between two consecutive heat stimuli (5-min interval). Following the application period, Bv8 was drained and heat stimuli were repeated at 5-min intervals. During the first heat stimulus after Bv8, the skin was still soaked with Bv8 solution, while all subsequent heat stimuli were applied after a 5-min SIF washout period. In part of the fibres, Bv8 was re-applied at higher concentration (as above) to the same fibre, provided the fibre's heat response had not been altered or had returned to a stable baseline. Ongoing and heat-induced neuronal activity was continuously recorded throughout the experiment.

For BV8 pre-treatment experiments, the skin-nerve flaps were placed at room temperature in either SIF solution (control) or SIF solution containing Bv8 at a concentration of $100 \mathrm{nmol} / \mathrm{L}$ for $90 \mathrm{~min}$ prior to the recording period. The recording procedure in these experiments was identical to the one described for acute Bv8 application except that no further Bv8 was applied after the conditioning incubation.

\section{Results}

\subsection{Stimulated CGRP release}

CGRP release experiments provided a first indication for BV8-induced heat sensitization of nociceptors.

In preliminary experiments, acute administration of Bv8 $(10,100$ and $310 \mathrm{nmol} / \mathrm{L})$, pre-applied to the hairy skin (for $5 \mathrm{~min}$ ) and then together with heat stimulation $\left(47^{\circ} \mathrm{C}\right)$, revealed that higher Bv8 concentrations elevate heat-induced CGRP release (Fig. 1A). Augmentation of the heat response was significant with $310 \mathrm{nmol} / \mathrm{L}$ Bv8 $(p<0.05$, Wilcoxon matched pairs comparing treated with untreated hind-paw skin of the same animal), $100 \mathrm{nmol} / \mathrm{L}$ appeared as effective (on average) but was not significant, and $10 \mathrm{nmol} / \mathrm{L}$ was ineffective to increase the heat-induced CGRP release.

One of the key receptors involved in cutaneous heat transduction and sensitization in the used 

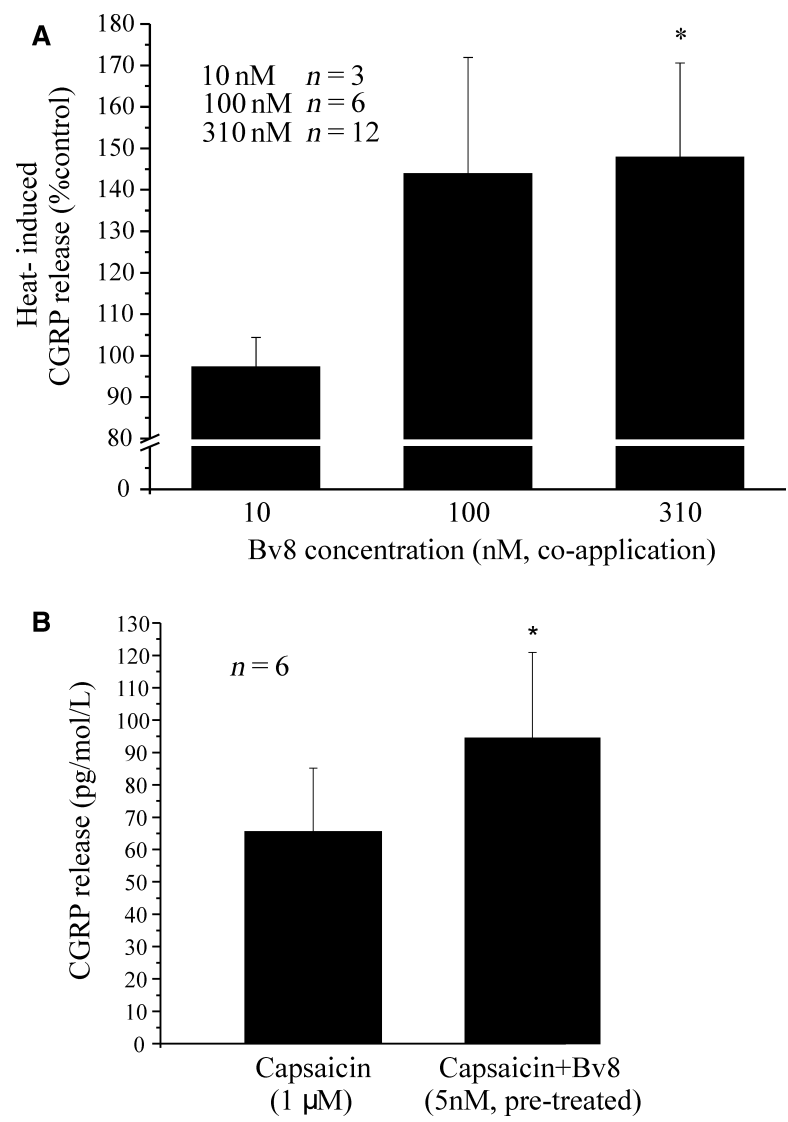

Figure 1 Bv8 effects on cutaneous CGRP release. (A) Heat-induced CGRP release in both males and females following acute Bv8 application (5-min incubation time). Bv8 was subsequently applied to the skin simultaneously with a heat stimulus $\left(47^{\circ} \mathrm{C}, 5 \mathrm{~min}\right)$. The heat-induced CGRP release in Bv8-treated skin flaps was normalized to the heat response of the control skin sample $\left(47^{\circ} \mathrm{C}\right.$, no Bv8) from the same animal and is presented as percentage of this control value. (B) Capsaicin-induced CGRP release following Bv8 pre-treatment. Skin flaps from female C57BL/6 were pre-incubated with either SIF or SIF + BV8 ( $5 \mathrm{nmol} / \mathrm{L}, 90 \mathrm{~min}$ ) and then analysed as above, stimulating CGRP release with capsaicin $(1 \mu \mathrm{mol} / \mathrm{L})$ in the third incubation step.

temperature range is the transient receptor potential channel [TRPV1, (Zimmermann et al., 2005; Petho et al., 2004)]. It is one of the main candidates for Bv8-induced heat sensitization. We, therefore, tested capsaicin (TRPVl agonist)-induced CGRP release in mouse skin flaps. Prolonged incubation (90 $\mathrm{min}$ ) of skin flaps with Bv8 ( $5 \mathrm{nmol} / \mathrm{L})$ prior to the experiment did not alter the baseline CGRP neurosecretion but significantly increased the capsaicin $(1 \mu \mathrm{mol} / \mathrm{L})$ induced CGRP release in females (Fig. 1B, $p=0.028$, Wilcoxon matched pairs as above) although not in males (male data not shown). Hence, prolonged pre-incubation with Bv8 requires much lower concentration than acute co-administration to induce nociceptor sensitization of TRPVl. Due to the sex
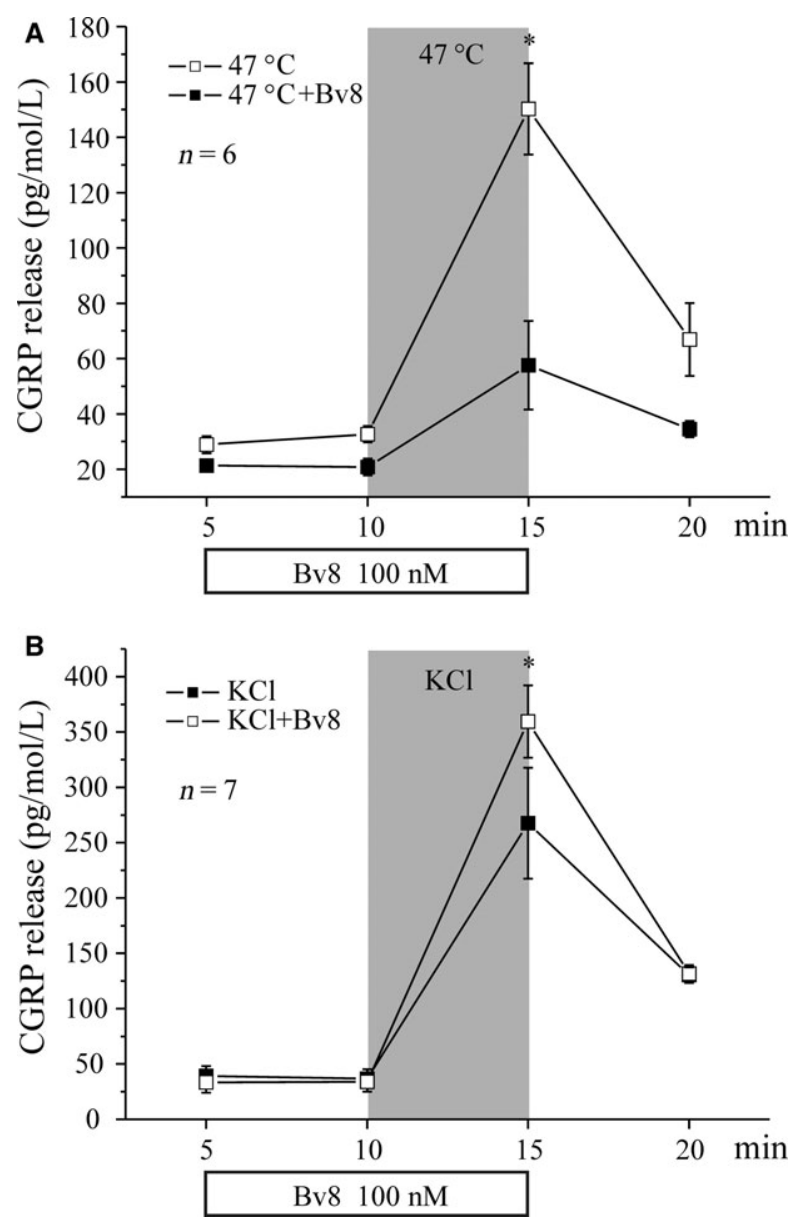

Figure 2 Bv8 effects on CGRP release from trigeminal ganglia. (A) Heatinduced CGRP release in skin flaps from female mice following acute BV8 (100 nmol/L) application for $5 \mathrm{~min}$. Bv8 was then applied to the skin simultaneously with heat stimulation $\left(47{ }^{\circ} \mathrm{C}, 5 \mathrm{~min}\right)$. (B) $\mathrm{KCl}(60 \mathrm{mmol} / \mathrm{L})$ induced CGRP release in skin flaps from female mice following acute Bv8 (100 nmol/L) application for $5 \mathrm{~min}$. Bv8 was then applied to the skin simultaneously with $\mathrm{KCl}$ stimulation (60 $\mathrm{mmol} / \mathrm{L}, 5 \mathrm{~min})$.

differences, all further experiments were performed in females.

Trigeminal ganglion (TG) neurons also express the Bv8 receptor PKR2 (Xiong et al., 2010) and are commonly used as a model of their peripheral or spinal terminals. Acute pretreatment with Bv8 (100 nmol/L for $5 \mathrm{~min}$ ) of TG yielded comparable results to the Bv8-induced heat sensitization seen in the skin (Fig. 2). While Bv8 by itself did not induce any CGRP release (Fig. 2A,B), it significantly augmented the heat-induced CGRP release in comparison to untreated contralateral ganglia (Fig. 2A, $p<0.001$, ANOVA repeated measures). The heat stimulus increased CGRP release by $2.6 \pm 0.4$ fold over baseline in untreated TG in comparison to a $4.7 \pm 0.4$ fold increase observed in Bv8-treated samples. 


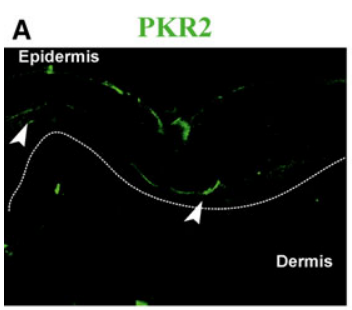

B

PKR2
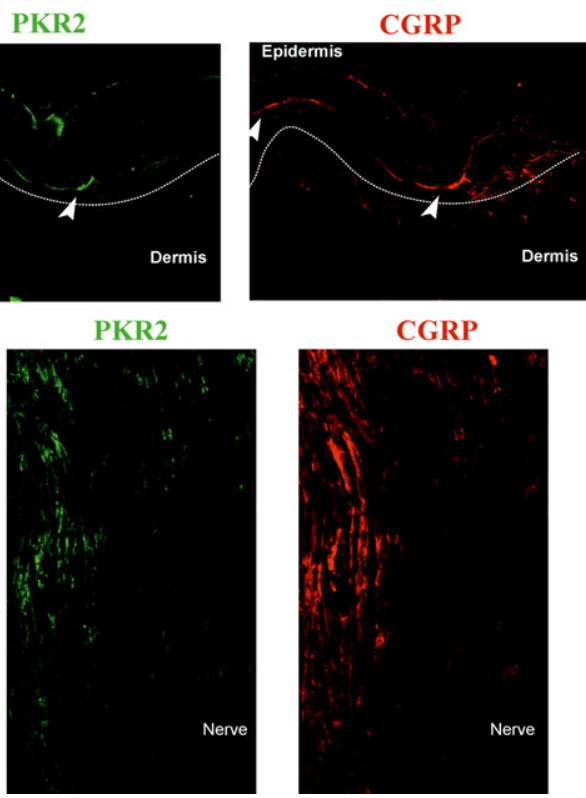

CGRP

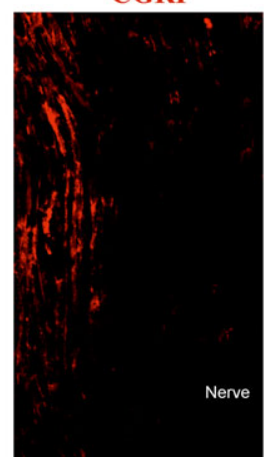

DAPI/PKR2/CGRP

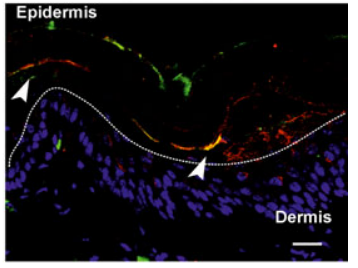

DAPI/PKR2/CGRP

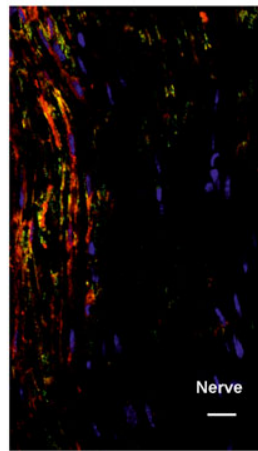

Figure 3 Co-localization of CGRP and prokineticin 2 in the skin and nerve. (A) Representative confocal images of mouse plantar skin sections immunostained for PKR2 (green) and CGRP (red). Double staining reveals co-localization of PKR2 with CGRP (yellow, pointed with an arrow) in some of the stained cells. Cell nuclei were counterstained with DAPI (blue). Dashed line represents the dermoepidermal junction. Scale bar - $20 \mu \mathrm{m}$. (B) Representative confocal images of longitudinally sliced sciatic nerve from the mouse immunostained for PKR2 (green) and CGRP (red). PKR2-green signal partly co-localized with CGRP positive fibres (yellow). Cell nuclei were counterstained with DAPI (blue). Scale bar - $20 \mu \mathrm{m}$.

In order to decide whether Bv8 effect only heat transduction or also general conductivity of nociceptive endings, Bv8 was tested together with a nonspecific depolarizing stimulus ( $\mathrm{KCl}, 60 \mathrm{mmol} / \mathrm{L})$. KCl-induced CGRP release was significantly elevated in Bv8-treated ganglia in comparison to untreated ones (Fig. 2B, $p<0.05$, ANOVA repeated measures), although to a lesser extent than that seen with heat stimulation. An $8 \pm 1.6$ fold increase over baseline was calculated with $\mathrm{KCl}$ alone in comparison to an $11 \pm 1.1$ fold increase with $\mathrm{KCl}$ and Bv8 co-application, which translates to $39 \%$ Bv8-induced increase of non-specific CGRP release in contrast to a sensitization of the heat response by $77 \%$. Thus, Bv8induced augmentation of CGRP release in response to heat is probably to the most part, although not exclusively through TRPVI sensitization.

\subsection{Co-localization of CGRP and prokineticin receptor 2}

Immunostaining revealed co-localization of the Bv8 mouse ortholog prokineticin receptor 2 (PKR2) and CGRP. PKR2 was found in part of the cells containing CGRP in plantar skin (Fig. 3A) and sciatic nerve (Fig. 3B). This further supports the notion that Bv8 can exert its peripheral effects through direct activation of peptidergic afferents.

\subsection{Electrophysiology}

Single-fibre recordings were obtained from mechano-heat sensitive (polymodal) primary afferent C-fibres with RFs in the isolated mouse skin. Acute as well as prolonged Bv8 applications were tested with regard to heat sensitization. In light of sex differences arising from the CGRP release experiments only females were used for the electrophysiological measurements. A lack of initial spontaneous activity in the fibres was a prerequisite for further testing.

In acute Bv8 application experiments (5-min application during the recording), a low concentration of Bv8 $(10 \mathrm{nmol} / \mathrm{L})$ augmented the heatinduced spike discharge (Fig. 4A). An enhanced rise of the discharge rate with increasing temperature, an increased peak discharge and enhanced afterdischarge during the passive cooling period signified this sensitization to heat. The averaged heat response following Bv8 application (10 nmol/L) was greater in comparison to the one prior to application (Fig. 4D, $p<0.05$, ANOVA, LSD post hoc test). Repetitive heat stimuli during the washout of Bv8 showed a slow and gradual reversal of the heat sensitization that wore off over 20 min (Fig. 4A,D). Application of higher Bv8 concentrations (30 and $100 \mathrm{nmol} / \mathrm{L}$ ) did not yield a significant increase of the heat responsiveness in the tested fibres (Fig. 4B-D), but 

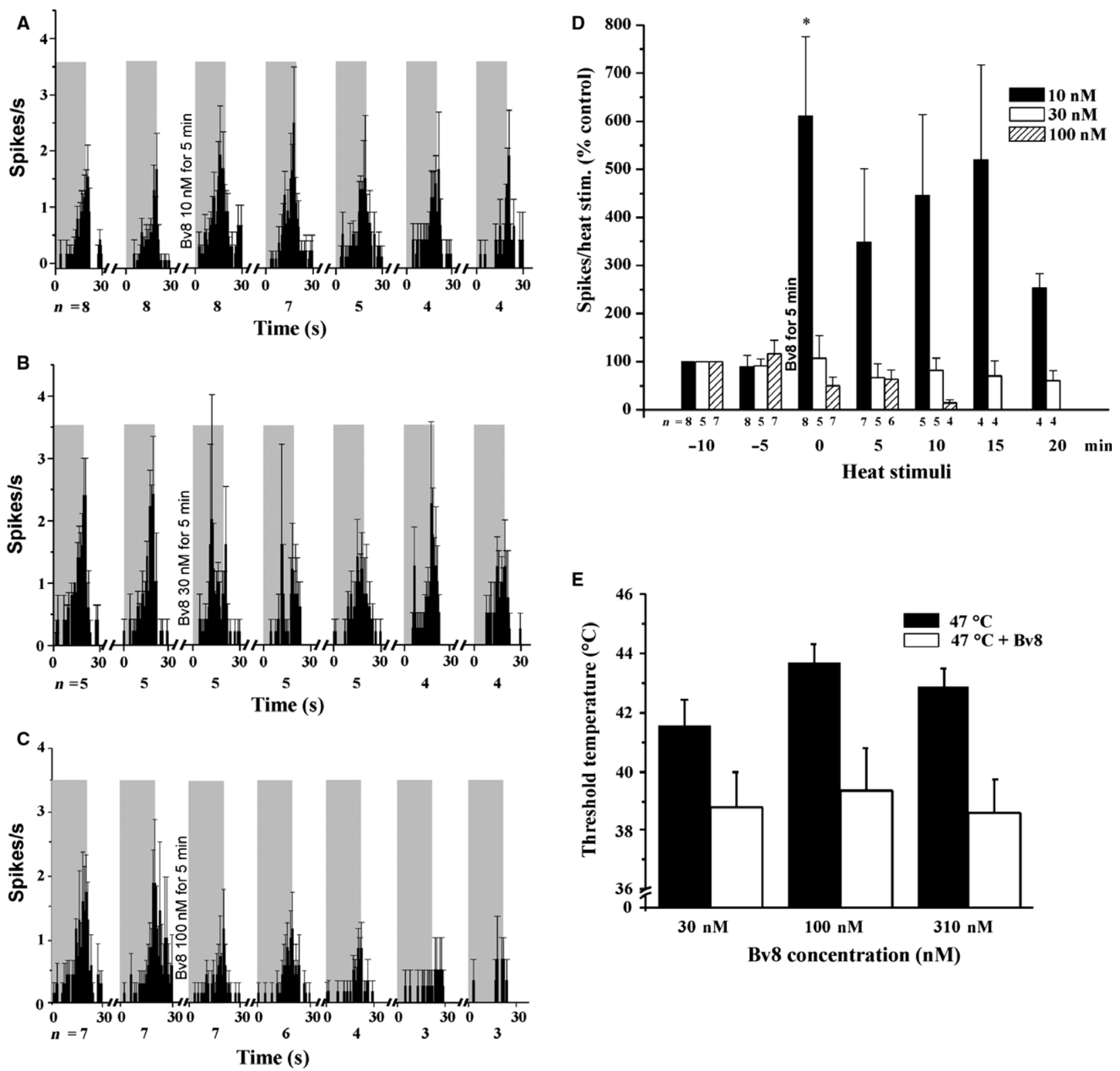

Figure 4 Heat-induced discharge in unmyelinated cutaneous fibres following Bv8 acute application. (A) Amplified heat responses in heat sensitive unmyelinated fibres following acute application of Bv8 at low concentrations (10 nmol/L, 5 min between consecutive heat stimuli) are shown. Heatinduced discharge (during the 20-s heat ramp) is highlighted in grey. Not in all fibres the complete length of the protocol could be executed. Thus, the number of averaged fibres from $(n)$ is specified for each stimulation. (B, C) Heat-induced ongoing activity following acute Bv8 application (as in A) at higher concentrations (30, $100 \mathrm{nmol} / \mathrm{L}$, respectively). (D) Total heat-induced discharge after acute application of Bv8. Heat responsiveness is represented as total number of spikes per heat stimulus (20-s ramp of $32^{\circ}-48{ }^{\circ} \mathrm{C}$ ), and response values for each concentration are normalized to the reciprocal values during the first heat stimulation (prior to Bv8 application). (E) Heat thresholds at two consecutive heat stimuli prior to and following acute application of Bv8 at different concentrations ( $30 \mathrm{nmol} / \mathrm{L} / 100 \mathrm{nmol} / \mathrm{L} / 310 \mathrm{nmol} / \mathrm{L}, 5 \mathrm{~min}$ between consecutive heat stimuli).

reduced the heat threshold in $4.3 \pm 0.2{ }^{\circ} \mathrm{C}$ for $100 \mathrm{mmol} / \mathrm{L}$ and $4.3 \pm 0.1^{\circ} \mathrm{C}$ for $310 \mathrm{mmol} / \mathrm{L}$ (Fig. 4E, $p<0.05$ and $p=0.067$, respectively, Wilcoxon matched pairs). The decreasing fibre number (n) in all tested fibres during repetitive stimulation could be attributed either to complete desensitization of the stimulus-induced response or to fibres' death.

Experiments with prolonged Bv8 pre-treatment of the whole isolated skin flap prior to recording, mimicking CGRP release trials (see above), confirmed 
that Bv8 induces heat sensitization. This Bv8 pretreatment lead to augmented heat responses (Fig. 5A). The peak discharge rates during the first heat ramp were greater by a factor of 2.7 (Fig. 5A, $p<0.05$, ANOVA, LSD post hoc test) and 2.6 during the second heat stimulus (Fig. 5B, $p=0.06$, ANOVA, LSD post hoc test) as compared with the untreated control population of C-fibres, and the rise in heatinduced discharge rate with temperature was obviously much steeper in pre-treated than naïve units. Despite the similarity in results, the pre-treatment in electrophysiological experiments was performed using a higher concentration of $100 \mathrm{nmol} / \mathrm{L} \mathrm{Bv} 8$ (for $90 \mathrm{~min}$ ) than that used for CGRP release trials. This was considered reasonable because of the long and unknown interval between the end of the pre-treatment period and the finding and testing of an appropriate single fibre which, in fact, required $70 \pm 9$ min on average (range 30-120 min). During this long washout period, any acute desensitizing Bv8 effect of the high concentration (see above) could be considered to be worn off.

Upon repeated heat stimuli, the heat threshold of cutaneous C-fibres shows a normal tendency to rise, due to desensitization/adaptation (Hoffmann et al., 2008). While the heat thresholds of untreated fibres following repeated heat stimulation displayed this mild desensitization (a $2.4{ }^{\circ} \mathrm{C}$ increase in heat threshold between 1 st and 2nd heat stimulus, $p=0.06$, Wilcoxon matched pairs), the (lower) heat thresholds of Bv8 pre-treated fibres became even lower with stimulus repetition (Fig. 4C), so that the heat threshold upon the second heat stimulation was now dramatically lower in Bv8 pre-treated fibres than in untreated ones $(p<0.05$, Mann-Whitney $U$-test). Thus, Bv8 appears to have induced not only a sensitization to heat but also an enhanced 'sensitizability', revealed by a second noxious heat stimulus.

\section{Discussion}

Our results demonstrate that Bv8 sensitizes cutaneous nociceptors to heat. Bv8 increases heatinduced CGRP release in both skin flaps and trigeminal ganglia, elevates heat-induced discharge in single cutaneous sensory afferents and lowers their thermal threshold. The fact that: (1) PKR2 co-localizes with CGRP in epidermal layers and sciatic nerves; (2) Bv8 application to the skin induces instant augmentation of heat-induced CGRP release; and (3) Bv8 sensitizes the heat responses of single sensory fibres indicates that Bv8 acts through direct activation of nociceptors in the skin. Together with Bv8's augmentation of
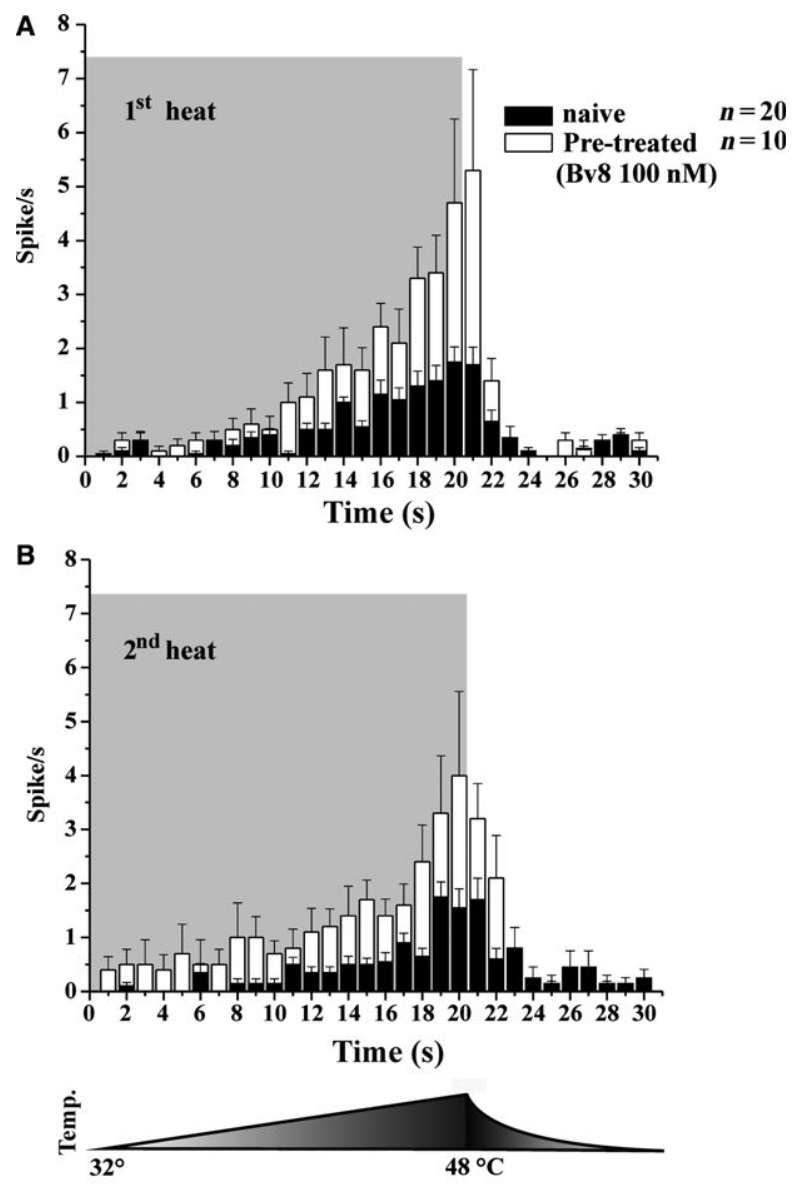

C

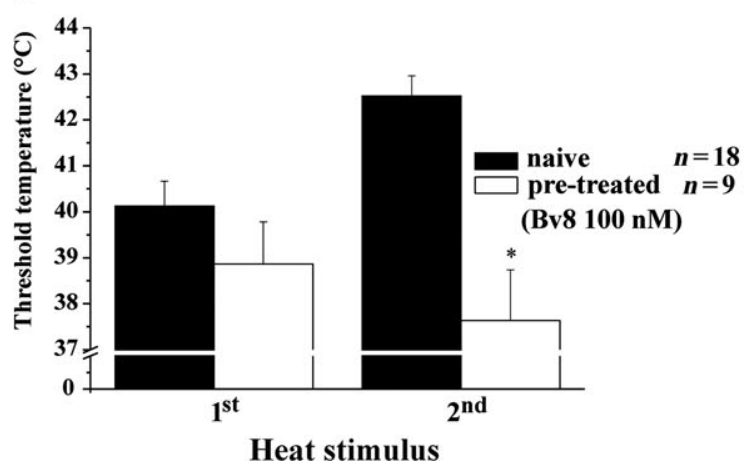

Figure 5 Heat-induced discharge in unmyelinated cutaneous fibres following pre-treatment with Bv8. (A, B) Heat-induced ongoing discharge. Probes were incubated with Bv8 (100 nmol/L) 90 min prior to experiment onset. Two successive heat stimuli (A and B, respectively, highlighted in grey) at 5-min interval were applied and ongoing discharge (spikes/s) was recorded. (C) Bv8-induced heat threshold oscillations. Heat thresholds at two consecutive heat stimuli following Bv8 pretreatment (100 nmol/L, 90 min prior to experiment onset) is depicted.

capsaicin-mediated CGRP release in skin flaps, these results imply that the activation mechanisms is, at least to some extent, TRPVI mediated. While $\mathrm{KCl}-$ 
induced CGRP release revealed a TRPVI-unspecific component, the fact that there was no significant activation by Bv8 alone (no direct CGRP release from skin flaps or spontaneous discharge in singlefibre measurements prior to heat stimulus) promotes the idea of a predominantly TRPVI-dependent heat sensitization mechanisms.

These results dovetail previously published data showing Bv8-induced hyperalgesia in mice and concomitant up-regulation of $\mathrm{Ca}^{2+}$ currents in DRGs. Our data provide the missing link between behavioural testing of referred pain and measurements of altered soma activity. In addition, a direct and possibly TRPVl-mediated effect of Bv8 on cutaneous nerve endings further corroborates the previous hypothesis that the initial response phase to cutaneous injection of Bv8 is due to peripheral sensitization. Our results do not shed further light regarding possible central sensitization mechanisms of the second response phase.

Our CGRP release experiments suggest that the sensitizing effect Bv8 exerts on peripheral nerve endings is restricted to females. While we found no supportive evidence to this in existing literature on Bv8 (due to the fact that there is mostly no mention of animal sex used for experimentation), we have previously observed differential plasma extravasation and neuronal heat sensitivity in response to invasive treatment in C57BL/6 male and female mice (personal observations). These differential responses were $\mathrm{PKC}_{\varepsilon}$ and progesterone mediated, which relate to the fact that in response to $\mathrm{Bv} 8$ a $\mathrm{PKC}_{\varepsilon}$-mediated sensitization of TRPVI in DRGs has been reported.

Both acute application onto the skin in in vitro physiology and prolonged pretreatment of the tissue with Bv8 in CGRP release experiments induced heat sensitization of cutaneous nerves, although with differential effective concentrations. Our experience with these two methods has showed that often different concentrations are needed for achieving comparable effectiveness. Methodological differences which might contribute to these discrepancies could be the sensitivity of the methods (single-fibre electrophysiology is sensitive to changes on a single-fibre level, while in CGRP release, activation of a large number of fibres is needed for a detectible level), the accessibility of Bv8 to the tissue (topical application directly onto the RF of a single fibre might be more effective in reaching the target zone than random circulation of Bv8 in the reaction tube), or time differences in the measured parameter (a higher Bv8 concentration would be required to induce the relatively time-consuming CGRP release from a high number of cutaneous fibres, in comparison to rapid augmentation of spontaneous heat induced firing in a single fibre). An additional reason for the differences in concentrations might be that the determination of effecting concentrations for CGRP release from skin flaps (Fig. 1A) was performed on a males-females mixed population, while parallel single-fibre electrophysiology was performed on female mice only. This might have shifted the concentration needed for reaching significance in CGRP release experiments to a higher level. Furthermore, CGRP release experiment showed that pretreatment of the skin with Bv8 is more efficient (i.e. requires lower concentrations) in inducing heat sensitization than acute application. Prolonged pretreatment with Bv8 might activate processes in an additive manner leading to a cumulated sensitization, explaining the lower effective concentrations.

In conclusion, Bv8 is a potent heat sensitizing agent, lowering threshold and increasing heat response at nanomolar concentrations. Further research concerning the involvement of prokineticin receptors in the development of thermal hyperalgesia (such as following neuropathy or inflammation) could reveal a valuable target for alleviating drugs.

\section{Acknowledgement}

The authors thank Birgit Vogler for excellent technical assistance.

\section{Author contributions}

T. Hoffmann performed the single-fibre recordings and CGRP release experiments and wrote the manuscript. L. Negri provided the Bv8 for the experiments and supervised the immunostaining experiments, D. Maftei and R. Lattanzi performed and analysed the immunostaining experiments and P. Reeh supervised single-fibre recordings and CGRP release experiments, as well as co-wrote the article. All authors discussed the results and approved the manuscript.

\section{References}

Averbeck, B., Reeh, P.W. (2001). Interactions of inflammatory mediators stimulating release of calcitonin gene-related peptide, substance $P$ and prostaglandin $E(2)$ from isolated rat skin. Neuropharmacology 40, 416-423.

Bretag, A.H. (1969). Synthetic interstitial fluid for isolated mammalian tissue. Life Sci 8, 319-329.

Cheng, M.Y., Bullock, C.M., Li, C., Lee, A.G., Bermak, J.C., Belluzzi, J., Weaver, D.R., Leslie, F.M., Zhou, Q.Y. (2002). Prokineticin 2 transmits the behavioural circadian rhythm of the suprachiasmatic nucleus. Nature 417, 405-410.

Eberhardt, M., Hoffmann, T., Sauer, S.K., Messlinger, K., Reeh, P.W., Fischer, M.J. (2008). Calcitonin gene-related peptide release from intact isolated dorsal root and trigeminal ganglia. Neuropeptides 42 , 311-317. 
Hoffmann, T., Sauer, S.K., Horch, R.E., Reeh, P.W. (2008). Sensory transduction in peripheral nerve axons elicits ectopic action potentials. J Neurosci 28, 6281-6284.

Immke, D.C., Gavva, N.R. (2006). The TRPVl receptor and nociception. Semin Cell Dev Biol 17, 582-591.

Joubert, F.J., Strydom, D.J. (1980). Snake venom. The amino acid sequence of protein A from Dendroaspis polylepis polylepis (black mamba) venom. Hoppe Seylers Z Physiol Chem 361, 1787-1794.

LeCouter, J., Ferrara, N. (2003). EG-VEGF and Bv8. a novel family of tissue-selective mediators of angiogenesis, endothelial phenotype, and function. Trends Cardiovasc Med 13, 276-282.

LeCouter, J., Kowalski, J., Foster, J., Hass, P., Zhang, Z., Dillard-Telm, L., Frantz, G., Rangell, L., DeGuzman, L., Keller, G.A., Peale, F., Gurney, A., Hillan, K.J., Ferrara, N. (2001). Identification of an angiogenic mitogen selective for endocrine gland endothelium. Nature 412, 877-884.

LeCouter, J., Zlot, C., Tejada, M., Peale, F., Ferrara, N. (2004). Bv8 and endocrine gland-derived vascular endothelial growth factor stimulate hematopoiesis and hematopoietic cell mobilization. Proc Natl Acad Sci USA 101, 16813-16818.

Levine, J.D., Fields, H.L., Basbaum, A.I. (1993). Peptides and the primary afferent nociceptor. J Neurosci 13, 2273-2286.

Lin, D.C., Bullock, C.M., Ehlert, F.J., Chen, J.L., Tian, H., Zhou, Q.Y. (2002). Identification and molecular characterization of two closely related $G$ protein-coupled receptors activated by prokineticins/ endocrine gland vascular endothelial growth factor. J Biol Chem 277, 19276-19280.

Maftei, D., Marconi, V., Florenzano, F., Giancotti, L.A., Castelli, M., Moretti, S., Borsani, E., Rodella, L.F., Balboni, G., Luongo, L., Maione, S., Sacerdote, P., Negri, L., Lattanzi, R. (2014). Controlling the activation of the $\mathrm{Bv} 8 /$ prokineticin system reduces neuroinflammation and abolishes thermal and tactile hyperalgesia in neuropathic animals. Br J Pharmacol 171, 4850-4865.

Melchiorri, D., Bruno, V., Besong, G., Ngomba, R.T., Cuomo, L., De, B.A., Copani, A., Moschella, C., Storto, M., Nicoletti, F., Lepperdinger, G., Passarelli, F. (2001). The mammalian homologue of the novel peptide Bv8 is expressed in the central nervous system and supports neuronal survival by activating the MAP kinase/PI-3-kinase pathways. Eur J Neurosci 13, 1694-1702.

Mollay, C., Wechselberger, C., Mignogna, G., Negri, L., Melchiorri, P., Barra, D., Kreil, G. (1999). Bv8, a small protein from frog skin and its homologue from snake venom induce hyperalgesia in rats. Eur $J$ Pharmacol 374, 189-196.

Negri, L., Lattanzi, R., Giannini, E., Metere, A., Colucci, M., Barra, D., Kreil, G., Melchiorri, P. (2002). Nociceptive sensitization by the secretory protein Bv8. Br J Pharmacol 137, 1147-1154.

Negri, L., Lattanzi, R., Giannini, E., Colucci, M.A., Mignogna, G., Barra, D., Grohovaz, F., Codazzi, F., Kaiser, A., Kreil, G., Melchiorri, P.
(2005). Biological activities of Bv8 analogues. Br J Pharmacol 146, $625-632$.

Negri, L., Lattanzi, R., Giannini, E., Colucci, M., Margheriti, F., Melchiorri, P., Vellani, V., Tian, H., De, F.M., Porreca, F. (2006a). Impaired nociception and inflammatory pain sensation in mice lacking the prokineticin receptor PKRl: Focus on interaction between PKRl and the capsaicin receptor TRPVl in pain behavior. J Neurosci 26, 6716-6727.

Negri, L., Lattanzi, R., Giannini, E., Melchiorri, P. (2006b). Modulators of pain: Bv8 and prokineticins. Curr Neuropharmacol 4, 207-215.

Negri, L., Lattanzi, R., Giannini, E., Melchiorri, P. (2007). Bv8/ Prokineticin proteins and their receptors. Life Sci 81, 1103-1116.

Petho, G., Izydorczyk, I., Reeh, P.W. (2004). Effects of TRPVl receptor antagonists on stimulated iCGRP release from isolated skin of rats and TRPV1 mutant mice. Pain 109, 284-290.

Reeh, P.W. (1986). Sensory receptors in mammalian skin in an in vitro preparation. Neurosci Lett 66, 141-146.

Sauer, S.K., Schafer, D., Kress, M., Reeh, P.W. (1998). Stimulated prostaglandin E2 release from rat skin, in vitro. Life Sci 62, 20452055.

Sauer, S.K., Bove, G.M., Averbeck, B., Reeh, P.W. (1999). Rat peripheral nerve components release calcitonin gene-related peptide and prostaglandin E2 in response to noxious stimuli: Evidence that nervi nervorum are nociceptors. Neuroscience 92, 319-325.

Schmelz, M., Forster, C., Schmidt, R., Ringkamp, M., Handwerker, H.O., Torebjork, H.E. (1995). Delayed responses to electrical stimuli reflect C-fiber responsiveness in human microneurography. Exp Brain Res 104, 331-336.

Soga, T., Matsumoto, S., Oda, T., Saito, T., Hiyama, H., Takasaki, J., Kamohara, M., Ohishi, T., Matsushime, H., Furuichi, K. (2002). Molecular cloning and characterization of prokineticin receptors. Biochim Biophys Acta 1579, 173-179.

Vellani, V., Colucci, M., Lattanzi, R., Giannini, E., Negri, L., Melchiorri, P., McNaughton, P.A. (2006). Sensitization of transient receptor potential vanilloid 1 by the prokineticin receptor agonist Bv8. J Neurosci 26, 5109-5116.

Wechselberger, C., Puglisi, R., Engel, E., Lepperdinger, G., Boitani, C., Kreil, G. (1999). The mammalian homologues of frog Bv8 are mainly expressed in spermatocytes. FEBS Lett 462, 177-181.

Xiong, Y.C., Li, X.M., Wang, X.J., Liu, Y.Q., Qiu, F., Wu, D., Gan, Y.B., Wang, B.H., Hu, W.P. (2010). Prokineticin 2 suppresses GABAactivated current in rat primary sensory neurons. Neuropharmacology 59, 589-594.

Zimmermann, K., Leffler, A., Fischer, M.M., Messlinger, K., Nau, C., Reeh, P.W. (2005). The TRPV1/2/3 activator 2-aminoethoxydiphenyl borate sensitizes native nociceptive neurons to heat in wildtype but not TRPV1 deficient mice. Neuroscience 135, 1277-1284. 